\title{
The scope of a combined kidney failure-heart failure clinic
}

\author{
Authors: Racquel Lowe-Jones, Joey Junarta, Ahmad Salha, Bayiha Klaud Francheska, Lisa Anderson, Juan C Kaski \\ and Debasish Banerjee
}

\begin{abstract}
Aims
Chronic kidney disease in heart failure (CKD-HF) is common, with high mortality, poor quality of life (related to anaemia, fluid overload) and multiple clinical issues which require a multidisciplinary approach. The role of a combined nephrologycardiology clinic to manage CKD-HF patients is not known, which this study investigates.
\end{abstract}

\section{Methods}

This study examined the referral patterns, comorbidities, management issues and interventions in patients referred to a combined CKD-HF clinic in an inner-city tertiary care hospital. All data were collected as part of an audit for patients referred over 10 months and analysed using SPSS 25.

\section{Results}

A total of 69 patients were referred between March and December 2017; 60 patients attended a first visit; 33 a second visit, seven a third visit and one a fourth visit. Three patients died during this period. Clinical characteristics were: age $76 \pm 13$ (mean \pm SD) years, males $69 \%$, hypertensives $88 \%$, diabetics $53 \%$, dyslipidaemics in $70 \%$. The laboratory results were eGFR $33 \pm 12$ $\mathrm{mL} / \mathrm{min} / 1.73 \mathrm{~m}^{2}$, potassium $4.56 \pm 0.49 \mathrm{mmol} / \mathrm{L}$, creatinine $186 \pm 88$ $\mu \mathrm{mol} / \mathrm{L}$, haemoglobin $114 \pm 21 \mathrm{~g} / \mathrm{L}$, ferritin $223 \pm 232 \mu \mathrm{g} / \mathrm{L}$. On echocardiogram the ejection fraction was $45 \pm 14 \%$ with half the population below $50 \%$.

At presentation 57 patients were on beta blockers, 43 on angiotensin converting enzyme inhibitors /angiotensin-receptor blockers (ACEi/ARBs), 20 patients on mineralocorticoid receptor antagonists (MRA). Six patients were on maximum dose of ACEi/ ARBs and one patient on maximum dose of MRA. One patient had a pre-visit potassium of $5.6 \mathrm{mmol} / \mathrm{L}$. Twenty-six (45\%) patients had a haemoglobin $<110 \mathrm{~g} / \mathrm{dL}$. Twenty-two patients had a ferritin $<200 \mu \mathrm{g} / \mathrm{L}$.

In the first visit $55(79 \%)$ received advice on diet, salt and fluid restriction. ACEi/ARB was changed or started in 15 (22\%) patients. MRA dose was changed or started in seven (10\%) patients. Twenty-one patients received intravenous iron, one had a second infusion and 10 infusions on the day of appointment. Serum ferritin improved between first and second visit $(p=0.046)$. Cardiac interventions performed as result of their first visit included dobutamine stress test, cardiac magnetic resonance imaging and placement of a resynchronisation pacemaker.

Patient feedback included comments such as, 'oh great, you brought the heart doctor as well', and, 'one less appointment, that's always good'.

\section{Conclusion}

This study shows that the CKD-HF patients referred to the new clinic suffered from multiple morbidity, low kidney function, low haemoglobin and iron stores. The patients rarely received maximum dose ACEi/ARB or MRA though hyperkalaemia was rare.

This novel outpatient clinic was able to address multiple renalcardiac issues in the same visit with a collaborative approach between two disciplines, with good patient feedback.

\section{Conflict of interest statement}

None

\section{Acknowledgement}

Dr Racquel Lowe-Jones is an International Society of Nephrology and Kidney Research UK fellow.

Authors: St George's University Hospital NHS Foundation Trust, Tooting, UK 